\title{
What can we learn from neonates with COVID-19?
}

\author{
Tian-Tian Xiao ${ }^{1,2} \cdot$ Kai Yan $^{1,2} \cdot$ Lai-Shuan Wang ${ }^{1,2} \cdot$ Wen-Hao Zhou ${ }^{1,2}$
}

Received: 16 April 2020 / Accepted: 29 May 2020 / Published online: 10 June 2020

(c) Children's Hospital, Zhejiang University School of Medicine 2020

The coronavirus disease 2019 (COVID-19) caused by severe acute respiratory syndrome coronavirus 2 (SARS-CoV-2) has become a global pandemic. Studies on epidemiological and clinical characteristics of patients with COVID-19 have indicated that children are susceptible to SARS-CoV-2 [1-3]. To date, the largest study in China reported that over $90 \%$ of 2143 children with COVID-19 were asymptomatic, or had mild or moderate illness, and that $5.8 \%$ presented critical illness [3]. Although the data of neonates are limited and only six neonates with COVID-19 have been reported thus far, several important points should be addressed from these neonatal cases.

\section{What are the routes of transmission in the neonates?}

The person-to-person transmission of SARS-CoV-2 has been confirmed by epidemiological studies of cases associated with COVID-19 [4]. Three late-onset neonatal COVID-19 cases were caused by familial aggregation infection, consistent with studies on children and adults with COVID-19 [4, 5]. However, COVID-19 still occurred in three neonates on day 2 after birth, even though strict infection control and preventive procedures were implemented [6]. A recent study described that the elevated IgM antibodies to SARS-CoV-2 were detected in a neonate born to its affected mother at $2 \mathrm{~h}$ after birth and the level of IgM was still elevated at 14 days later, indicating the possibility of vertical transmission [7]. However, a previous study showed that there were no clinical findings suggestive of COVID-19 in 19 neonates born to infected mothers, and all samples, including nasopharyngeal

Wen-Hao Zhou

zhouwenhao@fudan.edu.cn

1 National Clinical Research Center for Children's Hospital of Fudan University, Shanghai, China

2 Department of Neonatology, Children's Hospital of Fudan University, 399 Wanyuan Road, Shanghai 201102, China and rectal swabs from neonates, amniotic fluid, cord blood, and breastmilk were detected negative for SARS-CoV-2 [8, 9]. Therefore, vertical transmission remains controversial. At present, no studies have demonstrated that there is live virus isolated from the stools of patients with COVID-19; therefore, fecal-oral transmission remains uncertain. Notably, one infected neonate presented vomiting and bloody stool with SARS-CoV-2 positive rectal swabs, indicating that the gastrointestinal system was involved in this neonate with COVID-19 [10].

\section{What are the clinical characteristics of neonates with COVID-19?}

Figure 1 shows the clinical history of six neonates with COVID-19. Four neonates presented mild illness. Two neonates with underlying conditions presented severe respiratory illness, consistent with the results obtained from children [3]. One neonate with atrial septal defect (ASD) presented critical severe pneumonia and cardiac dysfunction [11], whereas the other neonate born at a gestational age of $31+2$ weeks was diagnosed with neonatal respiratory distress syndrome (NRDS) and sepsis with an Enterobacter agglomerates positive blood culture [6]. All the neonates had favorable outcomes (Fig. 1). The most common symptoms were fever, tachypnea and vomiting. However, different from children and adults, no neonates presented cough. Respiratory, gastrointestinal and cardiovascular systems can be all involved in neonates with COVID-19. Compared with adults, six neonates presented normal white blood cell counts and one had lymphocytopenia [6, 10-12]. Although the National Health Commission of the People's Republic of China has recommended serological diagnosis criteria for COVID-19, the features of immune response in neonates remain unclear. Two recent studies described the elevated IgM and IgG antibodies to SARS-CoV-2 in the neonates born to mothers with COVID-19; yet all the neonates were negative for SARS-CoV-2, and the follow-up time of the serological test was too short [7, 13]. Therefore, 


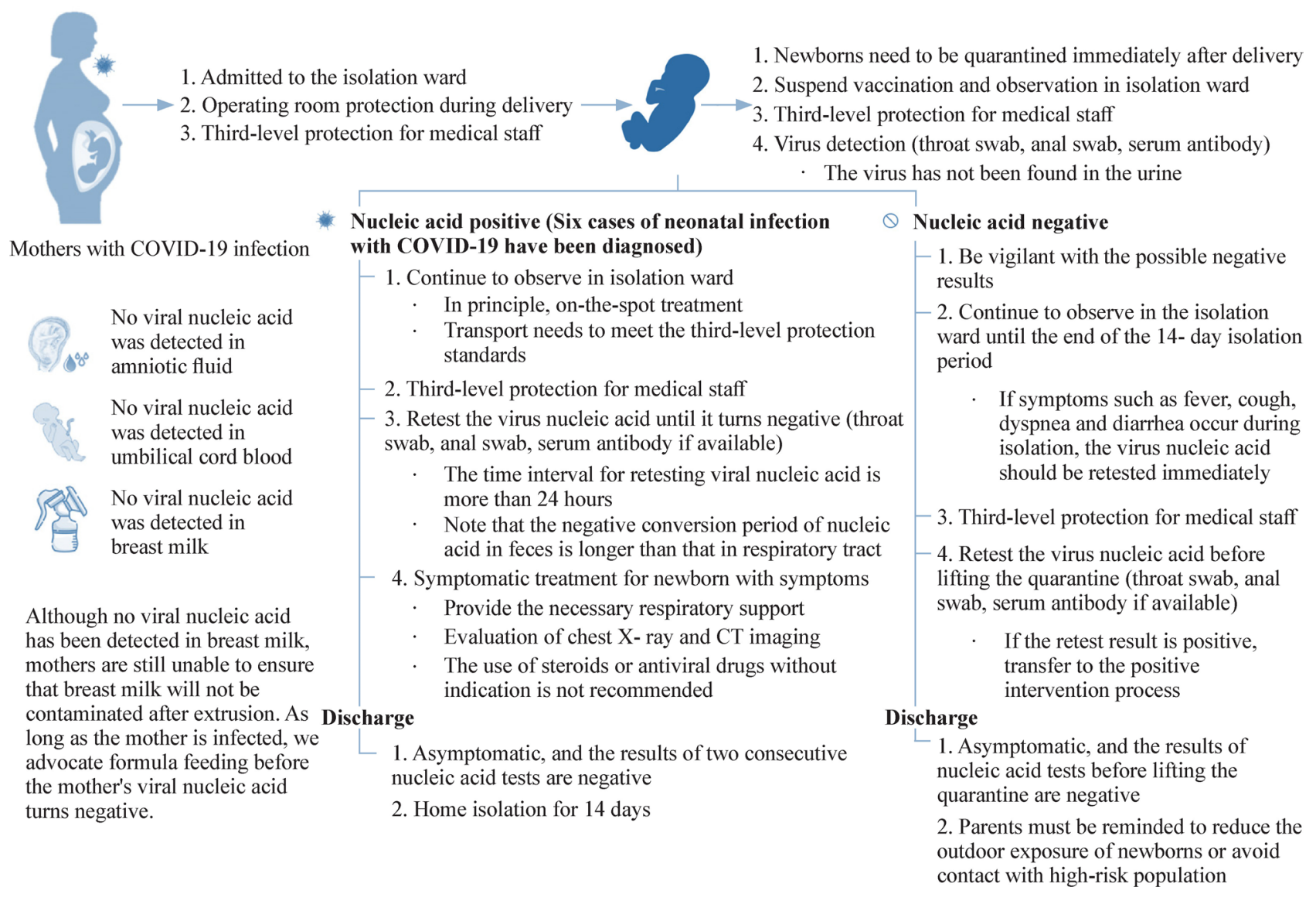

Fig. 1 The timeline and findings of six neonates infected with SARS-CoV-2

further studies should be initiated to investigate the dynamic immune response in neonates with COVID-19 or in neonates born to infected mothers. Also, typical imaging findings were observed in adults; yet radiological findings were non-specific in the six infected neonates $[6,10-12]$.

\section{How to manage the neonates with COVID-19?}

The flow diagram for managing neonates with COVID-19 is shown in COVID-19. Four affected neonates with mild symptoms were managed without intensive care. However, as stated above, one neonate with congenital heart disease (6.9 mm ASD) presented pneumonia and cardiac insufficiency; symptoms improved rapidly with inotropic therapy and fluid management in this neonate [11]. Another preterm infant with pneumonia, neonatal respiratory distress syndrome (NRDS), and sepsis was recovered with noninvasive ventilation support, caffeine and antibiotics [6]. These two cases provided a clue that although the symptomatic neonates with COVID-19 with underlying pulmonary diseases or congenital heart diseases received no antiviral therapy, they could improve rapidly after early and appropriate treatments. Moreover, the manifestations of neonatal COVID-19 are indistinguishable from NRDS, transient tachypnea, pneumonia, sepsis, etc. Therefore, it is important to investigate other etiologies if the clinical course of neonates with COVID-19 is not as expected and to protect those neonates with comorbidities including immunodeficiency disease, congenital heart disease, inherited metabolic disease, neuromuscular disorders, etc. Next, it is critical to decide discharge time from hospital. The current criterion is two consecutive results showing negative for SARS-CoV-2 using upper airway specimen (with at least a 24-h interval). However, the rectal swabs from two neonates remained positive for SARS-CoV-2 after nasopharyngeal swabs turned negative [10,12], consistent with the results obtained from the study in pediatric patients [14]. Therefore, the criterion may need to be revised based on the available data. Currently, no evidences have shown that the SARS-CoV-2 can be transmitted from neonates to other neonates or to caregivers or healthcare workers. Importantly, as the possibility of fecal-oral transmission exists, the appropriate education should be offered to parents with respect to hand hygiene and disinfection of children's excreta at home. 


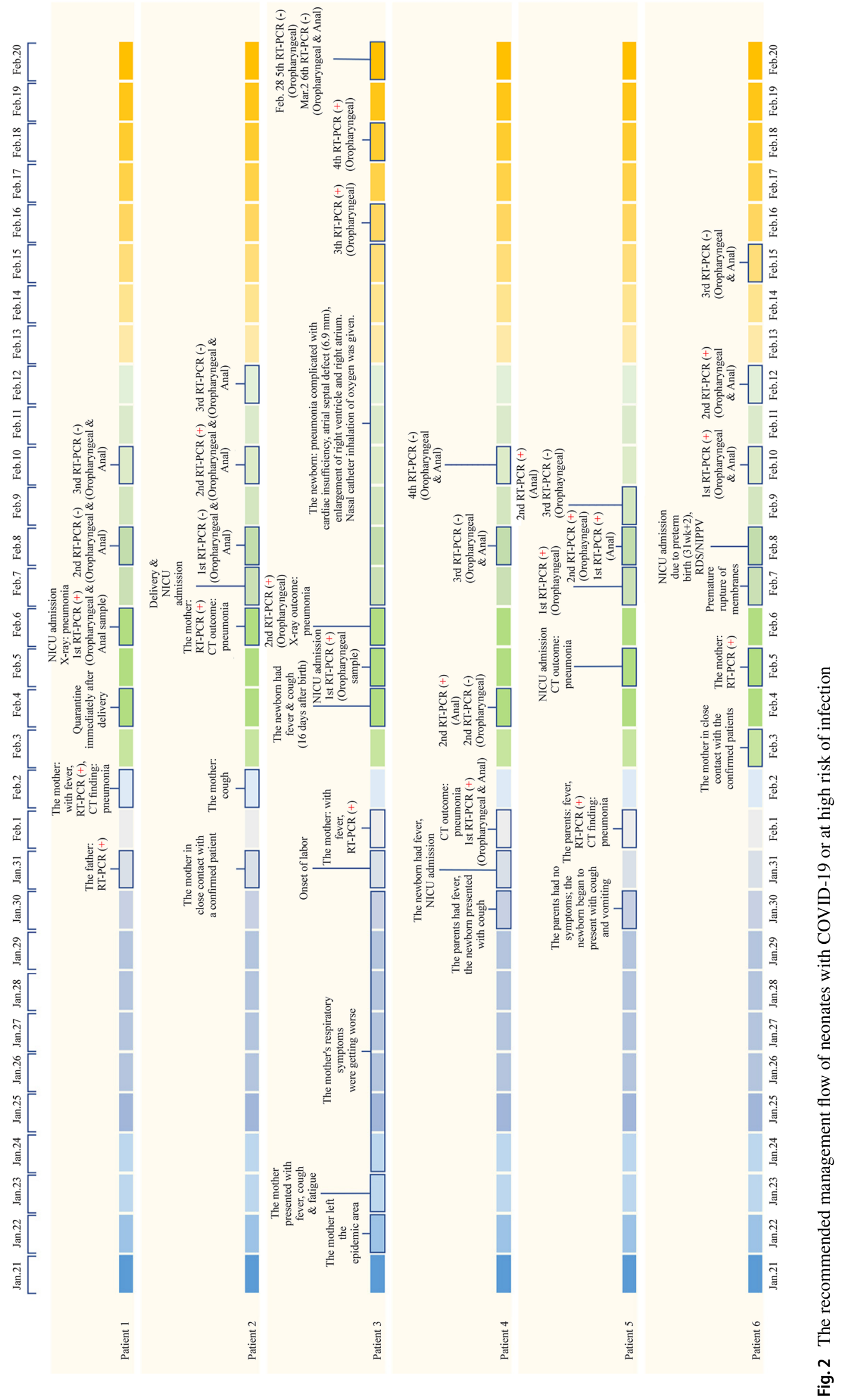




\section{How to manage the neonates born to mothers with COVID-19?}

Figure 2 shows the management flow for neonates born to mothers with COVID-19. As stated above, the familial cluster infection has been confirmed but vertical transmission remains uncertain. Thus, all neonates born to mothers with COVID-19 are at high risk of infection and should be separated from mothers, and fed by formula milk initially. The infected mothers with two consecutive negative tests for SARS-CoV-2 should be kept isolated for another 14 days, and then the infants can be breastfed by mothers. The isolated mothers should keep pumping to maintain breastmilk. Meanwhile, considering the virus may be excreted into the breastmilk of the infected mothers, donor milk and breastmilk can be considered for use after being screened for SARS-CoV-2. Family support should be offered in the case of maternal-neonatal separation and maternal depression. In the delivery room, to reduce the risk of the vertical transmission of SARS-CoV-2, delayed cord clamping (DCC) and mother-baby contact are also not recommended in China.

Author contributions XTT and YK contributed equally to this paper. XTT, YK wrote the first draft; WLS, ZWH contributed papers and guidance to initial submission. After review and request for more information, WLS, ZWH updated the document; all authors made final recommendations and edits. All authors approved the final version of the manuscript.

Funding None.

\section{Compliance with ethical standards}

Ethical approval Not required for this viewpoint.

Conflict of interest The authors have no conflicts of interest to declare.

\section{References}

1. Liu W, Zhang Q, Chen J, Xiang R, Song H, Shu S, et al. Detection of Covid-19 in children in early January 2020 in Wuhan, China. N Engl J Med. 2020;382:1370-1.
2. Pan A, Liu L, Wang C, Guo H, Hao X, Wang Q, et al. Association of public health interventions with the epidemiology of the COVID-19 outbreak in Wuhan, China. JAMA. 2020. https://doi. org/10.1001/jama.2020.6130.

3. Dong Y, Mo X, Hu Y, Qi X, Jiang F, Jiang Z, et al. Epidemiological characteristics of 2143 pediatric patients with 2019 coronavirus disease in China. Pediatrics. 2020. https://doi.org/10.1542/ peds.2020-0702.

4. Li Q, Guan X, Wu P, Wang X, Zhou L, Tong Y, et al. Early transmission dynamics in Wuhan, China, of novel coronavirus-infected pneumonia. N Engl J Med. 2020;382:1199-207.

5. Wei M, Yuan J, Liu Y, Fu T, Yu X, Zhang ZJ. Novel coronavirus infection in hospitalized infants under 1 year of age in China. JAMA. 2020. https://doi.org/10.1001/jama.2020.

6. Zeng L, Xia S, Yuan W, Yan K, Xiao F, Shao J, et al. Neonatal early-onset infection with SARS-CoV-2 in 33 neonates born to mothers with COVID-19 in Wuhan, China. JAMA Pediatr. 2020. https://doi.org/10.1001/jamapediatrics.2020.0878.

7. Dong L, Tian J, He S, Zhu C, Wang J, Liu C, et al. Possible vertical transmission of SARS-CoV-2 from an infected mother to her newborn. JAMA. 2020. https://doi.org/10.1001/ jama.2020.4621.

8. Chen H, Guo J, Wang C, Luo F, Yu X, Zhang W, et al. Clinical characteristics and intrauterine vertical transmission potential of COVID-19 infection in nine pregnant women: a retrospective review of medical records. Lancet. 2020;395:809-15.

9. Zhu H, Wang L, Fang C, Peng S, Zhang L, Chang G, et al. Clinical analysis of 10 neonates born to mothers with 2019-nCoV pneumonia. Transl Pediatr. 2020. https://doi.org/10.21037/ tp.2020.02.06.

10. Wang J, Wang D, Chen GC, Tao XW, Zeng LK. SARS-CoV-2 infection with gastrointestinal symptoms as the first manifestation in a neonate. Chin J Contemp Pediatr. 2020;22:211-4 (in Chinese).

11. Yu ZY, Xue W, Feng YJ, Bai QD, Jiang ML, Wang J, et al. Transfer diagnosis and treatment of a newborn with severe SARS-CoV-2 infection: a case report. Chin J Evid Based Pediatr. 2020;15:1-5 (in Chinese).

12. Zeng LK, Tao XW, Yuan WH, Wang J, Liu X, Liu ZS, et al. First case of neonate infected with novel coronavirus pneumonia in China. Chin J Pediatr. 2020. https://doi.org/10.3760/ cma.j.issn.0578-1310.2020.0009(in Chinese).

13. Zeng H, Xu C, Fan J, Tang Y, Deng Q, Zhang W, et al. Antibodies in infants born to mothers with COVID-19 pneumonia. JAMA. 2020. https://doi.org/10.1001/jama.2020.4861.

14. Xu Y, Li XF, Zhu B, Liang HY, Fang CX, Gong Y, et al. Characteristics of pediatric SARS-CoV-2 infection and potential evidence for persistent fecal viral shedding. Nat Med. 2020. https:// doi.org/10.1038/s41591-020-0817-4.

Publisher's Note Springer Nature remains neutral with regard to jurisdictional claims in published maps and institutional affiliations. 